\title{
Effect of Pr-Fe doping on dielectric and modulus properties of $\mathrm{SrTiO}_{3}$
}

\section{films}

Xiaofei Wang ${ }^{\mathrm{a}, c^{*}}$, Qiubo Hu ${ }^{\mathrm{b}}$, Guozhong Zang ${ }^{\mathrm{a}, \mathrm{c}}$, Chao Zhang ${ }^{\mathrm{a}, \mathrm{c}}$, Liben $\mathrm{Li}^{\mathrm{a}, \mathrm{c}}$

${ }^{a}$ School of Physics and Engineering, Henan University of Science and Technology, Luoyang 471023, PR China

${ }^{b}$ Department of Mathematics and Science, Luoyang Institute of Science and Technology, Luoyang 471023, PR China

${ }^{c}$ Luoyang Key Laboratory of Photonic and Electronic Materials, Luoyang 471023, PR China

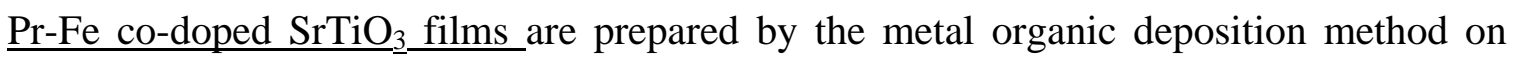
(111) $\mathrm{Pt} / \mathrm{Ti} / \mathrm{SiO}_{2} / \mathrm{Si}$ substrates. Their dielectric and electric modulus behaviors are investigated in the temperature range of $30 \sim 500^{\circ} \mathrm{C}$ with different measuring frequency of $10^{2} \sim 10^{6} \mathrm{~Hz}$. A typical ferroelectric relaxation phase transition is shown and its location shifts to the low temperature with the increase of Fe content. The imaginary parts of electric modulus $\mathrm{M}^{\prime \prime}$ exhibits the variable relaxation process with different $\mathrm{Fe}$ content, which is relation with OVs, PNRs and phase transition, and the possible causes of relaxation behavior dependence are analyzed in detail.

Keywords: Dielectric relaxation; Electric modulus; Metal organic deposition method PACS: 68.55.-a, 77.22.Gm, 77.55.+f

*Corresponding author at: School of Physics and Engineering, Henan University of Science and Technology, Luoyang, 471023, PR China. Tel.: +86 37964279206.

E-mail address: xfw628@haust.edu.cn (X.F. Wang). 


\section{Introduction}

Single-phase multiferroic materials are the substances possessing simultaneously ferroelectric and ferromagnetic properties, which can be used to convert the magnetic signals to electric responses or vice versa, and become excellent candidates for new generation memory devices, spintronics, sensors etc [1]. Among the best candidates for such applications are solid solutions of a ternary perovskite $\mathrm{PbFe}_{0.5} \mathrm{Ta}_{0.5} \mathrm{O}_{3}, \mathrm{BiFeO}_{3}$, $\mathrm{Lu}_{3} \mathrm{Fe}_{5} \mathrm{O}_{12}, \mathrm{BiMnO}_{3}, \mathrm{YMnO}_{3}$ and so on[2,3]. However, there remain several issues to be solved before it is practically applied in multiferroic devices operating at room temperature including inhomogeneous spin structure, small ferroelectric polarization, high leakage current and low Curie temperature [4]. Therefore, exploiting new materials that let magnetism and ferroelectricity coexist in a single phase above room temperature have become a hot spot [5].

$\mathrm{SrTiO}_{3}$ (STO), as a quantum paraelectric material, shows many interesting properties, including ferroelectric behavior, ferromagnetism, superconductivity etc., which is related to the lattice vibration, electric polarization, and the transportation of electrons in the matrix of the STO lattice [6-8]. With the development of the multiferroics, the coexistence of two or more properties in $\mathrm{SrTiO}_{3}$ will be received extensive concern in the future [7, 9]. Kim et al. [5] reported a room temperature multiferroic properties in $\left(\mathrm{Fe}_{\mathrm{x}}, \mathrm{Sr}_{1-\mathrm{x}}\right) \mathrm{TiO}_{3}$ thin films. Then the room temperature multiferroic properties have been further improved by Wang et al [10] using Pr-Fe co-doping methods in STO films, but its dielectric properties have not been paid sufficient attention. For STO materials, the 
dielectric anomalies are attributable to the doping of impurities such as oxygen vacancies (OVs), polar nanoregions (PNRs) and defect centers [11-15], which provides people with a method to have a better understanding of the movement mechanism of charged-defects and to adjust material properties. In this work, the dielectric properties and the electric modulus of multiferroic materials $\mathrm{Pr}-\mathrm{Fe}$ co-doped $\mathrm{SrTiO}_{3}$ films are studied. The variable relaxation processes are found for different Fe content, and the possible causes of which are analyzed and discussed.

\section{Experimental}

$\mathrm{Sr}_{0.975} \mathrm{Pr}_{0.025} \mathrm{Ti}_{1-\mathrm{x}} \mathrm{Fe}_{\mathrm{x}} \mathrm{O}_{3}(\mathrm{x}=0.1,0.2$ and 0.3$)$ films were fabricated by the metal organic deposition (MOD) method on (111) $\mathrm{Pt} / \mathrm{Ti} / \mathrm{SiO}_{2} / \mathrm{Si}$ substrates. Strontium acetate, tetrabutyl titanate, praseodymium oxide $\left(\operatorname{Pr}_{6} \mathrm{O}_{11}\right)$ and iron nitrate were used as the precursors for $\mathrm{Sr}, \mathrm{Ti}, \mathrm{Pr}$ and $\mathrm{Fe}$, respectively. Acetic acid, 2-methoxyethanol, and nitric acid were the solvent. The films were deposited by spin coating on (111) $\mathrm{Pt} / \mathrm{Ti} / \mathrm{SiO}_{2} / \mathrm{Si}$ substrates and were crystallized at $750{ }^{\circ} \mathrm{C}$ for $1 \mathrm{~h}$ in $\mathrm{O}_{2}$. The microstructure of the films was characterized by $\mathrm{X}$-ray diffraction (XRD, D/Max-RB) with $\mathrm{Cu} \mathrm{K \alpha}$ radiation. Morphology and chemical composition of the samples were studied by the scanning electron microscopy (SEM) and Energy-dispersive X-ray spectroscopy (EDX) using

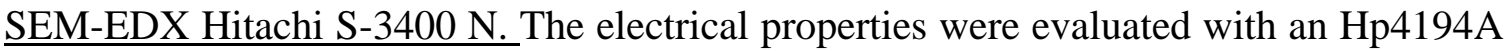
impedance/phase analyzer.

\section{Results and discussion}

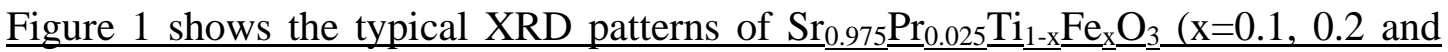


0.3) films at room temperature. These samples possess the polycrystalline structure of pure cubic perovskite phase with no additional peaks similar to the results of pure STO [17]. The surface morphology of Pr-Fe co-doped STO films detected by SEM is shown in the insets of Fig. 1(a)-(c). Three kinds of STO films are crystallized well and dense. The average grain sizes are about $100 \mathrm{~nm}$ for $\mathrm{x}=0.1$ sample and they gradually decrease with increasing Fe content. According to the results of EDX analysis, the nominal Fe content from 0.1 to 0.3 in our samples is actually $0.093,0.177,0.277$, respectively. In addition, the content of Prions is not detected due to the precision of the instrument.

Fig. 2(a), (b) and (c) show the variation of $\varepsilon^{\prime}$ and the loss $\tan \delta\left(\tan \delta=\frac{\varepsilon^{\prime \prime}}{\varepsilon}\right)$ with temperature at different frequencies for $\mathrm{Fe}=0.1,0.2$ and 0.3 samples, respectively. Here $\varepsilon^{\prime}$ and $\varepsilon^{\prime \prime}$ are the real and imaginary parts of the dielectric constant, respectively. For $\varepsilon^{\prime}$, its maximum value would decrease with the increase of frequency and corresponding peak temperature $\left(T_{p}\right)$ shifts up to higher temperatures, showing high frequency dispersion. This above-mentioned result is a typical relaxor behavior of the ferroelectric-paraelectric transition, whose position obviously shifts to lower temperature with increasing Fe ions. The same dielectric peaks were also shown in Pr-doped STO sample at a higher temperature $\left(240{ }^{\circ} \mathrm{C}\right)$ strongly, indicative of a ferroelectric-paraelectric transition, which may be the displacive character with Curie temperature of $\sim 118{ }^{\circ} \mathrm{C}[16]$. For $\tan \delta$, with increasing temperature its value increases stepwise and shows no obvious relaxation process. It turns out that $\tan \delta$ is deviating from the relaxation behaviors and reaches higher value ( over 100 at $500{ }^{\circ} \mathrm{C}$ ) due to the bigger leakage behavior that an extra 
conducting channel could be formed in the samples, which is relation with the coexistence of $\mathrm{Fe}^{2+}$ and $\mathrm{Fe}^{3+}$ ions. With increasing $\mathrm{Fe}$ content from 0 to 0.3 , the leakage current density of $\mathrm{Sr}_{0.975} \mathrm{Pr}_{0.025} \mathrm{Ti}_{1-\mathrm{x}} \mathrm{Fe}_{\mathrm{x}} \mathrm{O}_{3}$ films increased from the order of magnitude of $10^{-7}$ to $10^{-5} \mathrm{~A} / \mathrm{cm}^{2}$ when E increased to about $100 \mathrm{KV} / \mathrm{cm}[10,17]$.

To clarify the origin of relaxation behavior, modulus has been done for our samples. Modulus research is an important theory, formulated by Taher et al. [18], which studies charge transport processes in ion conductors and eliminates electrode polarization effect. The complex electric modulus $\left(\mathrm{M}^{*}\right)$ is calculated from the following equation and is defined as the reciprocal of the complex dielectric constant $\left(\varepsilon^{*}\right.$, here $\left.\varepsilon^{*}=\varepsilon^{\prime}+\mathrm{i} \varepsilon^{\prime \prime}\right)$

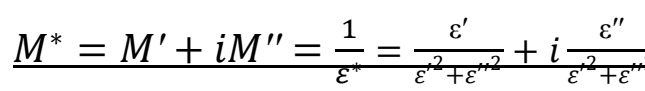

where $\mathrm{M}^{\prime}$ and $\mathrm{M}^{\prime \prime}$ are the real and imaginary parts of electric modulus, respectively. Figure 3 shows the variation of the imaginary part of the electric modulus $\left(\mathrm{M}^{\prime \prime}\right)$ as a function of temperatures at different frequency. A $\mathrm{M}^{\prime \prime}$ peak appears near $284^{\circ} \mathrm{C}$ in the $\mathrm{M}^{\prime \prime}$-temperature spectrum at $100 \mathrm{~Hz}$ and its location moves to higher temperature with the increase in frequency, which shows a typical relaxation behavior in $\mathrm{x}=0.1$ sample (as shown in Fig.3(a)). With the Fe ions increased to 0.2, two sets of relaxation peaks begin to appear at $99.8^{\circ} \mathrm{C}$ and $195.1^{\circ} \mathrm{C}$, respectively (as shown in Fig.3 (b)). In Fig. 3(c), one relaxation behavior is observed. These relaxation processes are labeled as regions I, II, III and IV for $\mathrm{x}=0.1,0.2$ and 0.3 samples, respectively. The maximum value of $\mathrm{M}^{\prime \prime}\left(\mathrm{M}^{\prime \prime}{ }_{\max }\right)$ happening is due to the interfacial polarization produced by the travelling charge carriers and pilling up at the interface of the two dielectrics. At a certain temperature, the 
influence of the applied field results in a free movement of the intrinsic immobilized charge carriers. The charge carriers hopping from one localized state to another may be represented by an analogous process of associated dipolar moment reorientation, and thus a dielectric relaxation occurs. In addition, as the relaxation peak shifts towards the higher temperature with increasing frequency, we consider that the charge carrier mobility increases and free charges accumulate at the interface within the sample. As a result, the relaxation time decreases and the peak shifts towards the higher temperature with increase in frequency. To clarify the origin of carrier hopping accounting for the present relaxation process, the detailed analysis is as follows:

According to the point defect relaxation theory, the peak height is in proportion to the concentration of relaxation units [19], the temperature variation of the characteristic frequency can be described by the Arrhenius relation. The relaxation time $(\tau)$ can be written as

$$
\tau=\tau_{0} \exp \left(U / k_{B} T\right)
$$

where $T$ is the absolute temperature, $\tau_{0}$ is the relaxation time at an infinite temperature,

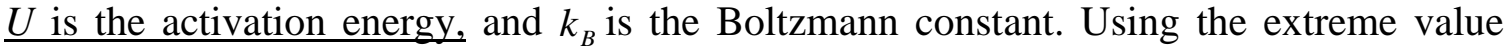
condition,

$$
\omega_{\max } \tau=1
$$

where angular frequency $\omega_{\max }=2 \pi f_{\max }\left(f_{\max }\right.$ is the frequency at which the maximum $\mathrm{M}^{\prime \prime}$ occurs). The relationship between the measuring temperature and peak frequency can $\underline{\text { be given by }}$ 


$$
\underline{\ln \left(2 \pi f_{\max }\right)=-U / k_{\mathrm{B}} \mathrm{T}}+\ln \tau_{0},
$$

according to Eq. (4), the peak frequency of $\mathrm{M}^{\prime \prime}$ at different temperature for $\mathrm{x}=0.1,0.2$ and 0.3 films are well fitted as shown in Fig. 4. These activation energies are listed in Fig.4 from the slope of the fitted straight line. In region I, the value is about $1.192 \mathrm{eV}$, which is close to the typical value $(1.0 \mathrm{eV})$ of activation energy for OVs in the perovskites or related oxide structures [20]. Paladino et al.[21] measured the activation energy for diffusion OVs in STO crystal to be $0.98-1.13 \mathrm{eV}$. Therefore, the relaxation peaks may be associated with the migration of $\mathrm{OVs}$ inside $\mathrm{Sr}_{0.975} \mathrm{Pr}_{0.025} \mathrm{Ti}_{0.9} \mathrm{Fe}_{0.1} \mathrm{O}_{3}$ film. Lu et al.[22] reported a broad relaxation peak of the dielectric loss above room temperature for pure STO ceramics, in which the activation energy was about $0.84 \mathrm{eV}$. Also the $\mathrm{Sr}_{0.975} \mathrm{Pr}_{0.025} \mathrm{TiO}_{3}$ ceramics showed a relaxation behavior which originated from the migration of OVs and the activation energy was about $0.85 \mathrm{eV}$ [23]. The activation energy for $\mathrm{x}=0.1$ sample at high temperature is slightly bigger than the ceramics mentioned above due to the relaxation parameters strongly affected by thermal history and grain boundaries $[24,25]$

With increasing Fe ions, in region II, the activation energy is about $1.441 \mathrm{eV}$ that is larger than the value of $x=0.1$ sample. We consider that the relaxation peaks may not be associated with the migration of OVs inside films because of the low-valence substitution of $\mathrm{Fe}^{2+} / \mathrm{Fe}^{3+}$ for $\mathrm{Ti}^{4+}$ ions, resulting in the increase of OVs. With the increase in OVs concentration and the formation of OVs clusters, the correlation among the OVs strengthens, and thus the movement of OVs becomes easier and the activation energy 
decreases [22], which is in contradiction with the change of activation energy from $x=0.1$ to 0.2 . It has been reported in the literature [23] that polar distortion may give rise to the relaxor ferroelectric behavior of Pr-doped STO due to the variable valence of Pr ions. Similarly, there should be more PNRs in $\mathrm{x}=0.2$ film as a result of the variable valence of Pr and Fe ions; the main contributors for this relaxation process (region II) may not be OVs, but PNRs.

In addition, the Arrhenius relation for the region III is not a straight line and the distinct deviation is very obvious. Grubbs et al.[26] ascribed the deviation to two Arrhenius segments, and the linear regions with different slopes suggested the different relaxation mechanisms in the corresponding regions. If the reduced electric modulus is plotted, $\mathrm{M}^{\prime \prime} / \mathrm{M}^{\prime \prime}{ }_{\max }$ as a function of $\mathrm{T}_{\mathrm{p}} / \mathrm{T}$, as shown in Fig. 5, we can see that the curves for the region III cannot merge into one master peak and the widened peaks at high frequencies, imply that there may be a different mechanism with the components from both long range and localized relaxation[27]. On second thoughts, similar to the results of $\mathrm{CaCu}_{3} \mathrm{Ti}_{4} \mathrm{O}_{12}$ by Zhang and Tang [28], the relaxation is possible to be related to polaron relaxation. According to the fitted curves by applying the following Mott's variable range hopping (VRH) relation

$$
f_{\max }=f_{0} \exp \left[-\left(\mathrm{T}_{0} / \mathrm{T}_{\mathrm{p}}\right)^{1 / 4}\right]
$$

where $f_{0}$ is the eigenfrequency which has a meaning of the inverse minimum relaxation

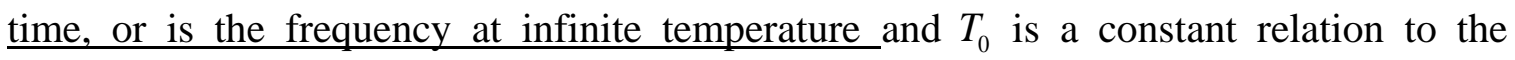


activation energy. From Fig. 6, the plot of $\ln f_{\max }$ vs $T_{P}^{-1 / 4}$, actually produces nonlinear behavior, which cannot be qualitatively explained in the frame of the polaronic model. In Fig.4. the activation energies from the slope of the theoretical fitting of the Arrhenius relation are $U_{\mathrm{III}}=1.159 \mathrm{eV}$ above $T \mathrm{c}$ and $U_{\mathrm{III}}=1.909 \mathrm{eV}$ below $T \mathrm{c}$. These values from 1.159 to $1.909 \mathrm{eV}$ are found to have a dielectric transition at about $264{ }^{\circ} \mathrm{C}$ corresponding to the results of Figure 2(b). Capacitance dispersion is significant in the temperature range in which the contributions of the two conduction mechanisms are comparable to each other. The higher frequency region may be due to the grain-grain boundary, meanwhile the lower frequency region is interpreted as the formation and migration of ions at the surface or electrode/crystal interfacial polarization [29]. To further confirm the results, Figure 7 shows the electric modulus spectrum $\left(\mathrm{M}^{\prime} \mathrm{vs}^{\prime \prime}\right)$ of the $\mathrm{Sr}_{0.975} \mathrm{Pr}_{0.025} \mathrm{Ti}_{0.8} \mathrm{Fe}_{0.2} \mathrm{O}_{3}$ at different temperature. The deformed semi-circles with their centers lying over the real axis indicate the distributions of relaxation time which support the non-Debye type of relaxation in the materials [30]. Due to the limitation of the experimental set-up, the electric modulus properties with higher or lower frequency cannot be obtained at present in our sample. Although the Cole-Cole figure shows only $1 / 4$ circles, it can be seen that the low frequency shrinkage is changed into high frequency. This further proves that there are two kinds of relaxation mechanisms in the vicinity of the phase transition.

Finally, in region IV, the activation energies are $U_{\mathrm{IV}}=1.567 \mathrm{eV}$ above $T \mathrm{c}$ and $U_{\mathrm{IV}}=$ $1.199 \mathrm{eV}$ below $T \mathrm{c}$, which show the similar relaxation behavior to region III. In short, 
with the increase of $\mathrm{Fe}$ ions, the numbers of the relaxation behaviors increase and decrease alternatively. We consider that when Fe content is 0.1 , the relaxation process mainly caused by OVs begins to appear (region I). With the increase of Fe ions from 0.1 to 0.3 , the relaxation process near the phase transition gradually moves towards the low temperature, and covers one by one the relaxation process caused by OVs (region I) and PRNs (region II). This work can regulate the electrical property STO films by PrFe co-doping methods and then facilitate practical applications.

\section{Conclusions}

In summary, $\mathrm{Sr}_{0.975} \mathrm{Pr}_{0.025} \mathrm{Ti}_{1-\mathrm{x}} \mathrm{Fe}_{\mathrm{x}} \mathrm{O}_{3}(\mathrm{x}=0.1,0.2$ and 0.3$)$ films are fabricated by MOD method. The location of the typical ferroelectric relaxation phase transition shifts to low temperature with the increase of Fe content. The variation of imaginary part of $\mathrm{M}^{*}$ with temperatures at different frequencies exhibits the different relaxation process for Pr-Fe co-doped STO samples, which are related with OVs, phase transition and PNRs. The process is analyzed in detail by Arrhenius diagram, Cole-Cole relation, modulus master curve and VRH relation. The fact that the incorporation of Fe ions leading to the changes of the internal defects of the films has a great influence on the dielectric properties of the films, which could be beneficial for understanding the movement mechanism of charged-defects and thus get a little closer to actual applications in microwave devices. 


\section{Acknowledgements}

This work was supported by the National Science Foundation (Grant Nos. 11504090), the National Science Foundation of Henan province (Grant Nos. 162300410089) and the cultivation of innovation fund of Henan University of Science and Technology (Grant no. 2014ZCX016). 


\section{References}

[1] J. Wang, J.B. Neaton, H. Zheng, V. Nagarajan, S.B. Ogale, B. Liu, D. Viehland, V. Vaithyanathan, D.G. Schlom, U.V. Waghmare, N.A. Spaldin, K.M. Rabe, M. Wuttig, R. Ramesh, Science 299 (2003) 1719.

[2] I.P. Raevski, V.V. Titov, M.A. Malitskaya, E.V. Eremin, S.P. Kubrin, A.V. Blazhevich, H. Chen, C.C. Chou, S.I. Raevskaya, I.N. Zakharchenko, D.A. Sarychev, S.I. Shevtsova, J Mate Sci 49 (2014) 6459.

[3] T. Choi, Y. Horibe, H.T. Yi, Y.J. Choi, W.D. Wu, S.W. Cheong, Nature Mater. 9 (2010) 253.

[4] J.F. Scott, NPG Asia Materials, 5 (2013) e72.

[5] K.T. Kim, C.Kim, S.P. Fang, Y.K. Yoona, Appl. Phys. Lett. 105 (2014) 102903.

[6] M. Choi, F. Oba, I. Tanaka, Phys. Rev. let. 103 (2009) 185502.

[7] J. He, X.M. Lu, W.L. Zhu, Y.Y. Hou, R.X. Ti, F.Z. Huang, X.L. Lu, T.T. Xu, J. Su, J.S. Zhu, Appl. Phys. Lett. 107 (2015) 012409.

[8] M. Choi, F. Oba, I. Tanaka, Phys. Rev. let. 103 (2009) 185502.

[9] H.S. Kim, L. Bi, G.F. Dionne, C.A. Rossb, Appl. Phys. Lett. 93 (2008) 092506.

[10] X.F. Wang, G.Z. Zang, Q.B. Hu, C. Zhang, S.J. Lv, L.B. Li, Mater Lett. 185 (2016) $545-548$.

[11]J. Bouwma, K.J. De Vries, A.J. Burggraaf, Phys. Status Solidi A 35 (1976) 281.

[12]Z. Yu, C. Ang, L.E. Cross, Appl. Phys. Lett. 74 (1999) 3044-3046. 
[13]C. Ang, Z. Yu, Appl. Phys. Lett. 90 (2007) 202903.

[14]C. Ang, J.F. Scott, Z. Yu, H. Ledbetter, J.L. Baptista, Phys. Rev. B 59 (1999) 6661.

[15]C. Ang, Z. Yu, Appl. Phys. Lett. 88 (2006) 162902.

[16]A. Durán, E. Martínez, J.A. Díaz, J.M. Siqueiros, J. Appl. Phys. 97 (2005) 104109.

[17] X.F. Wang, X.M. Lu, Y.Y. Weng, W. Cai, X.B. Wu, Y.F. Liu, F.Z. Huang, J.S. Zhu, Solid State Commu, 150 (2010) 267-270.

[18]Y.B. Taher, A. Oueslati, K. Khirouni, M. Gargouri, J Clust Sci 26 (2015) 1655-1669.

[19]S. Nowick, B.S. Berry, Anelastic Relaxation in Crystalline Solids (Academic, New York, 1972).

[20]J. F. Scott, Ferroelectric Memories Springer, Berlin, 2000.

[21]A.E. Paladino, J. Am. Ceram. Soc. 48 (1965)476.

[22]X.F Wang, X.M Lu, C. Zhang, X.B. Wu, W. Cai, S. Peng, H.F. Bo, Y. Kan, F.Z. Huang, J.S. Zhu, J. Appl. Phys. 107 (2010) 114101.

[23]X.F. Wang, Q.B. Hu, L.B. Li, X.M. Lu, J. Appl. Phys. 112 (2012) 044106.

[24]F. Li, Z. H. Zhou, J. Wang, J. Appl. Phys. 100 (2006) 034106.

[25] H.Y. Wang, L. Chen, H. Meng, X.M. Xiong, J.X. Zhang, Phys. Status Solidi B 246 (2009) 2392-2395

[26] R.K. Grubbs, E.L. Venturini, P.G. Clem, J.J. Richardson, B.A. Tuttle, G.A. Samara, Phys. Rev. B,72 (2005) 104111.

[27] Y.J. Wong, J. Hassan, M. Hashim, J. Alloys Compd. 571 (2013) 138-144.

[28] L. Zhang, Z. Tang, Phys. Rev. B, 70 (2004) 174306. 
[29] J.H. Park, J.S. Bae, B.C. Choi, J.H. Jeong, J. Phys. D: Appl. Phys. 40 (2007) 579583.

[30] R. Ranjan, R. Kumar, N. Kumar, B. Behera, R.N.P. Choudhary, J. Alloys Compd. 509 (2011) 6388-6394. 


\section{Figure captions}

FIG. 1. X-ray diffraction patterns of the polycrystalline $\mathrm{Sr}_{0.975} \operatorname{Pr}_{0.025} \mathrm{Ti}_{\mathrm{x}} \mathrm{Fe}_{1-\mathrm{x}} \mathrm{O}_{3}$ film at room temperature. The insets (a), (b) and (c) show SEM topography images of $x=0.1,0.2$ and 0.3 , respectively.

FIG. 2. Temperature dependence of $\varepsilon^{\prime}$ and tan $\delta$ at various frequencies of $10^{2}, 10^{2.5}, 10^{3}$, $10^{3.5}, 10^{4}, 10^{4.5}, 10^{5}, 10^{5.5}$, and $10^{6} \mathrm{~Hz}$ for $\operatorname{Sr}_{0.975} \operatorname{Pr}_{0.025} \mathrm{Ti}_{\mathrm{x}} \mathrm{Fe}_{1-\mathrm{x}} \mathrm{O}_{3}$ films (a) $\mathrm{x}=0.1$; (b) $\mathrm{x}=0.2$; and $(\mathrm{c}) \mathrm{x}=0.3$.

FIG. 3. M" as a function of temperature at various frequencies of $10^{2}, 10^{2.5}, 10^{3}, 10^{3.5}, 10^{4}$, $10^{4.5}, 10^{5}, 10^{5.5}$, and $10^{6} \mathrm{~Hz}$ for Pr-Fe co-doped $\mathrm{SrTiO}_{3}$ films with different Fe content.

FIG. 4. The plot between $\ln \left(2 \pi f_{\max }\right)$ and $1 / \mathrm{k}_{\mathrm{B}} \mathrm{T}$ of $\mathrm{Pr}-\mathrm{Fe}$ co-doped $\mathrm{SrTiO}_{3}$ films $(\mathrm{x}=0.1$, 0.2 and 0.3 ) samples according to the Arrhenius law. The solid line is the Arrhenius fitting.

FIG. 5. M"/M" ${ }_{\max }$ vs $\mathrm{T}_{\mathrm{p}} / \mathrm{T}$ curve of $\mathrm{Sr}_{0.975} \mathrm{Pr}_{0.025} \mathrm{Ti}_{0.8} \mathrm{Fe}_{0.2} \mathrm{O}_{3}$ at various frequencies.

FIG. 6. Peak frequency of $M "$ as a function of $100 / \mathrm{T}_{\mathrm{P}}{ }^{1 / 4}$ for region III and IV.

FIG. 7. The imaginary parts as a function of real parts of the electric modulus. 


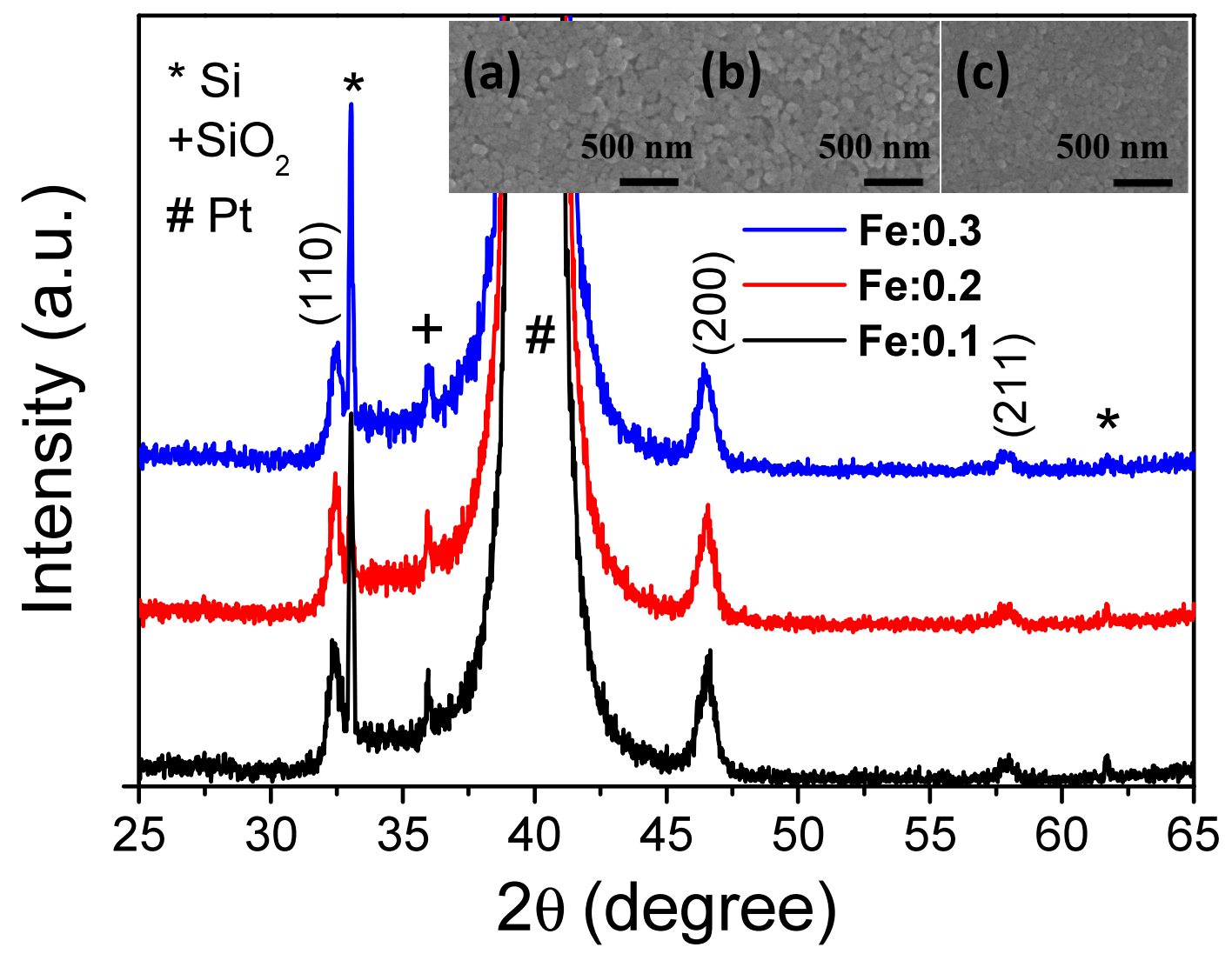

Figure 1 


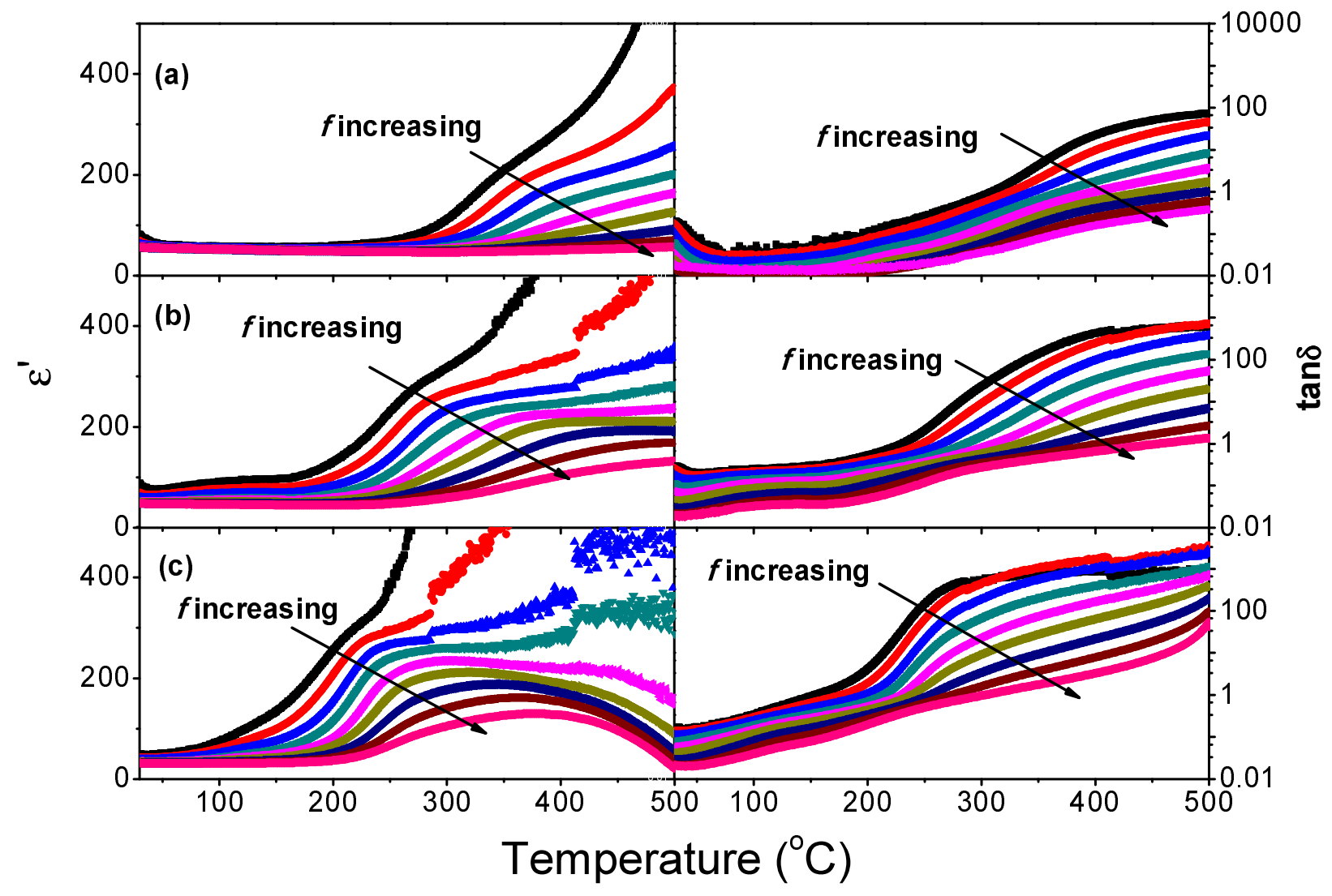

Figure 2 


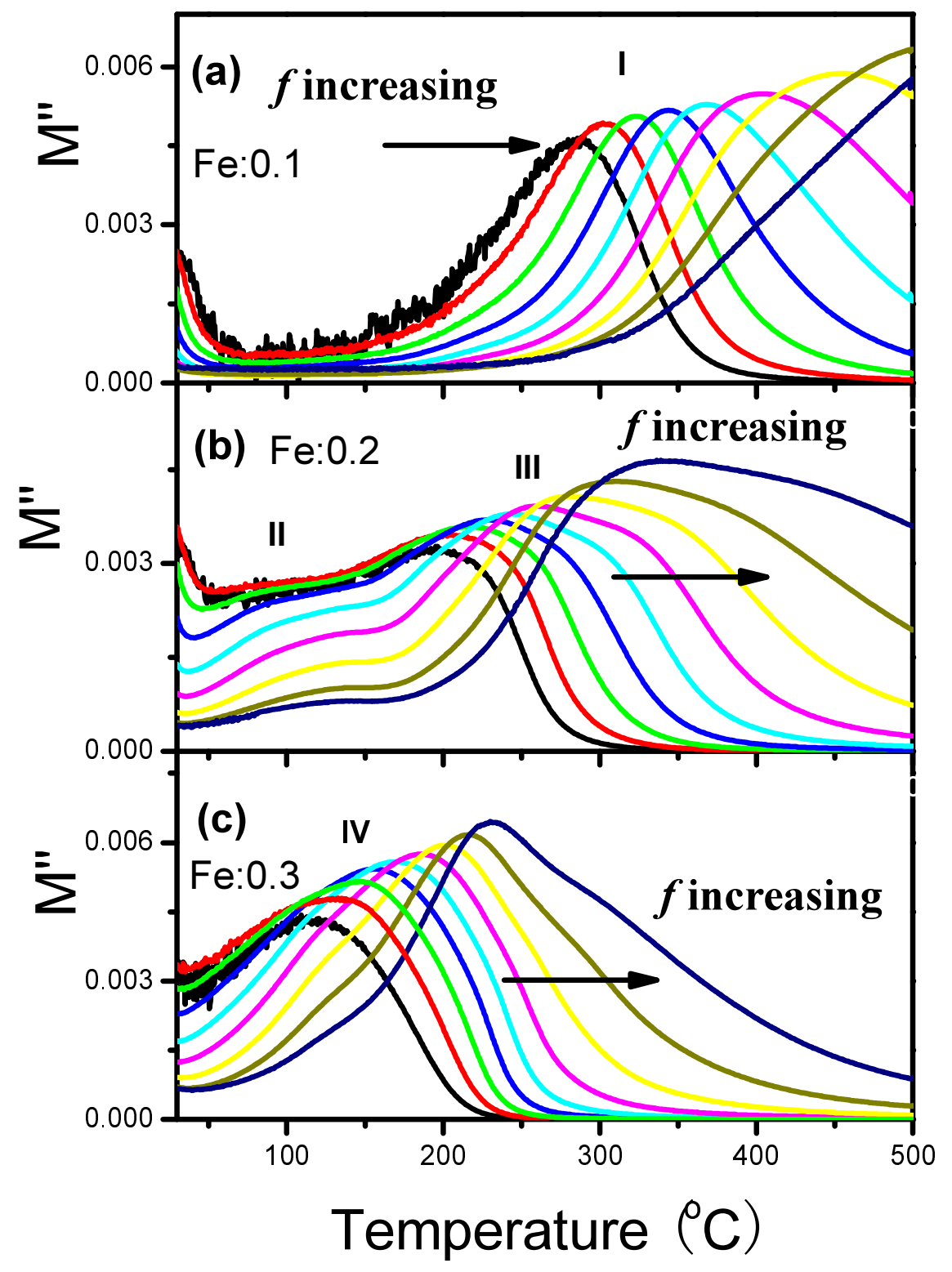

Figure 3 
Figure 4

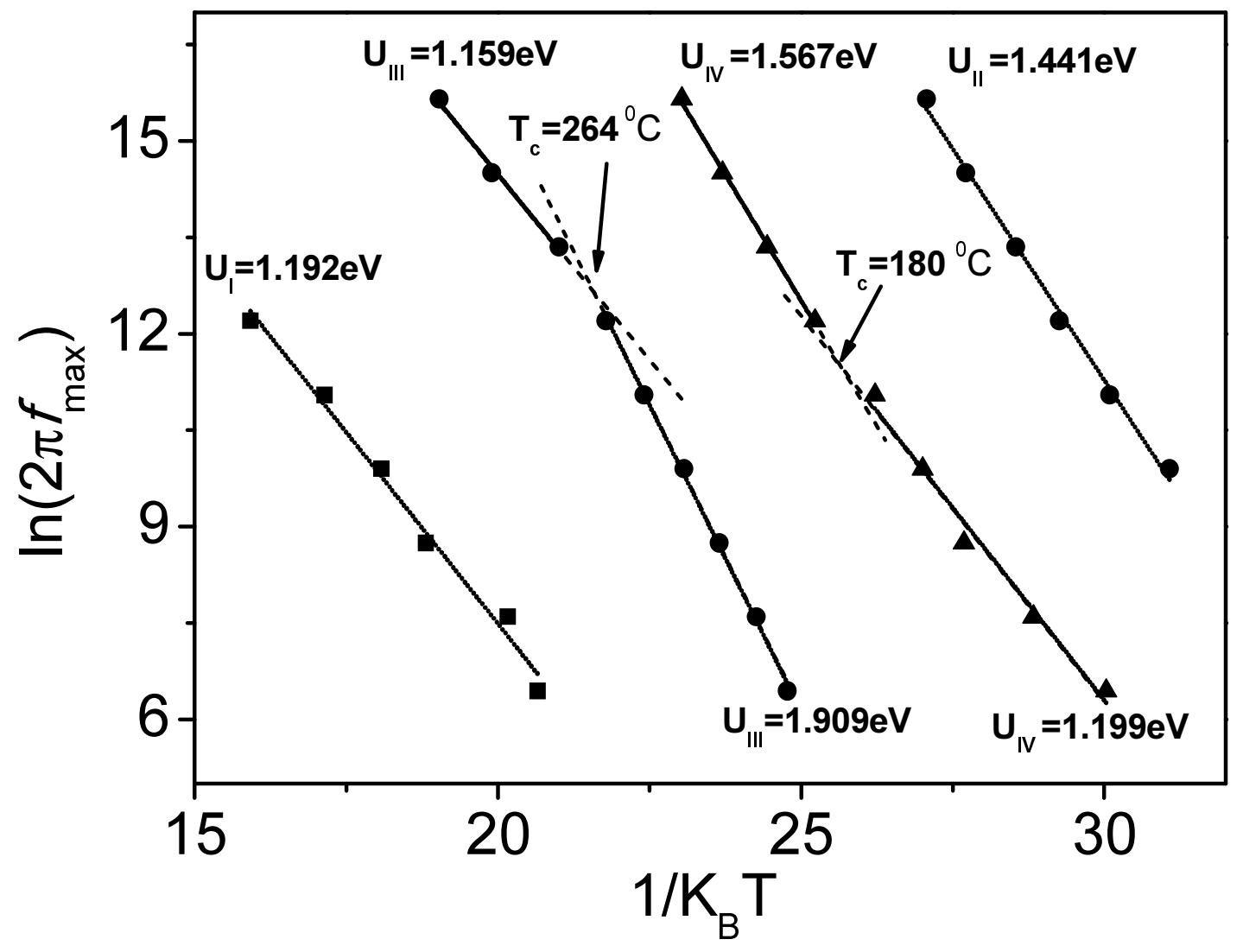

Figure 4 
Figure 5

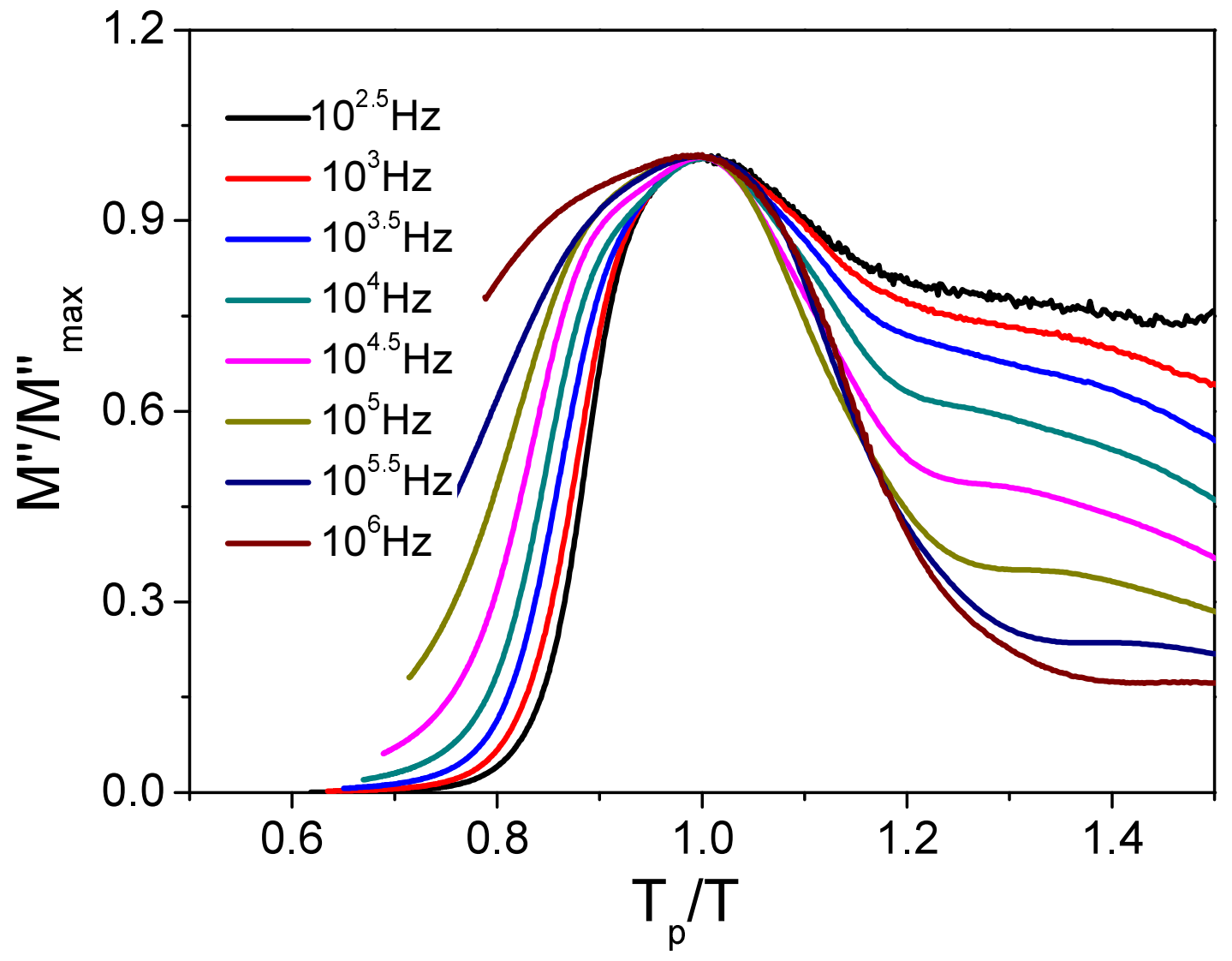

Figure 5 
Figure 6

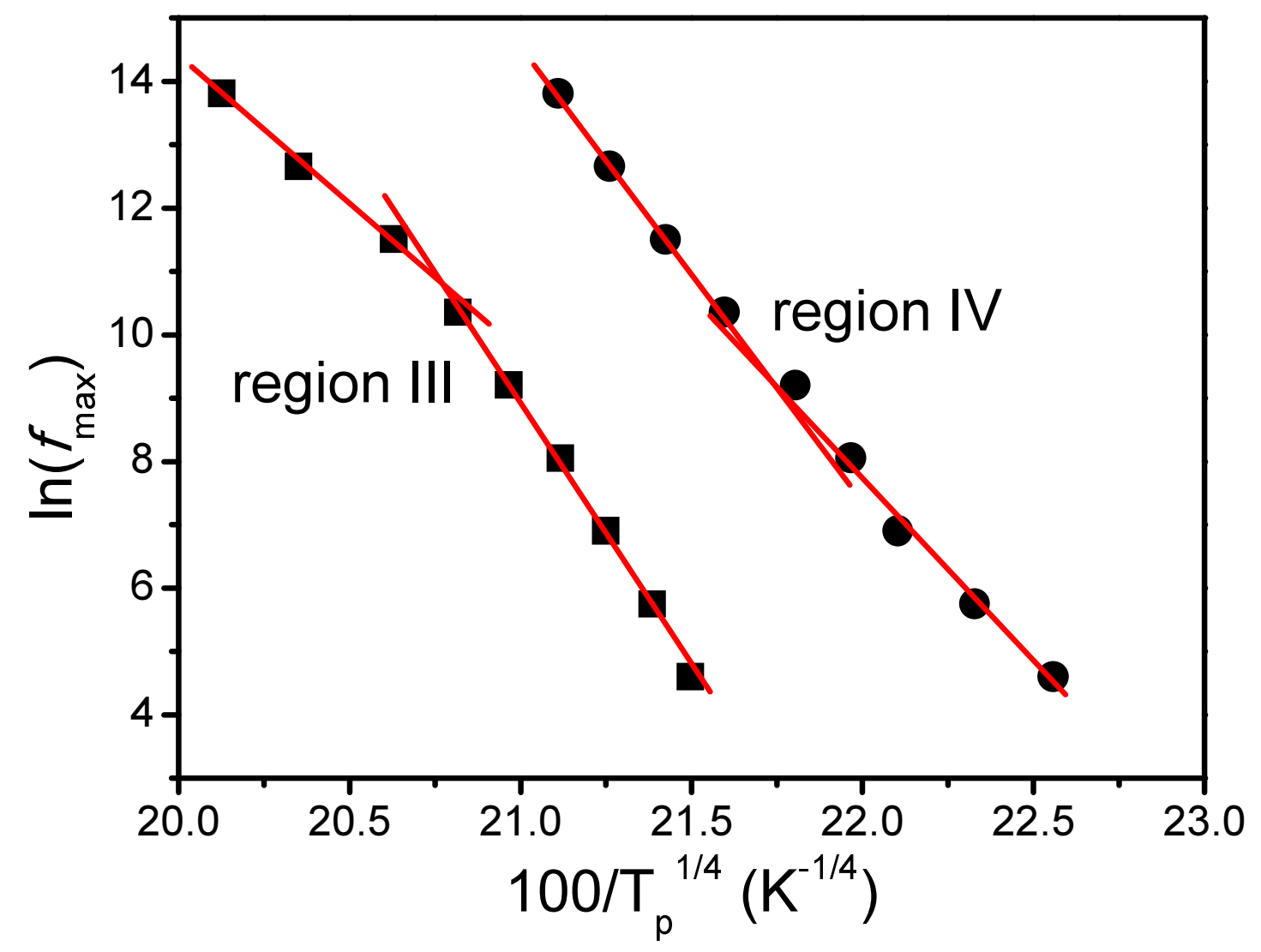

Figure 6 


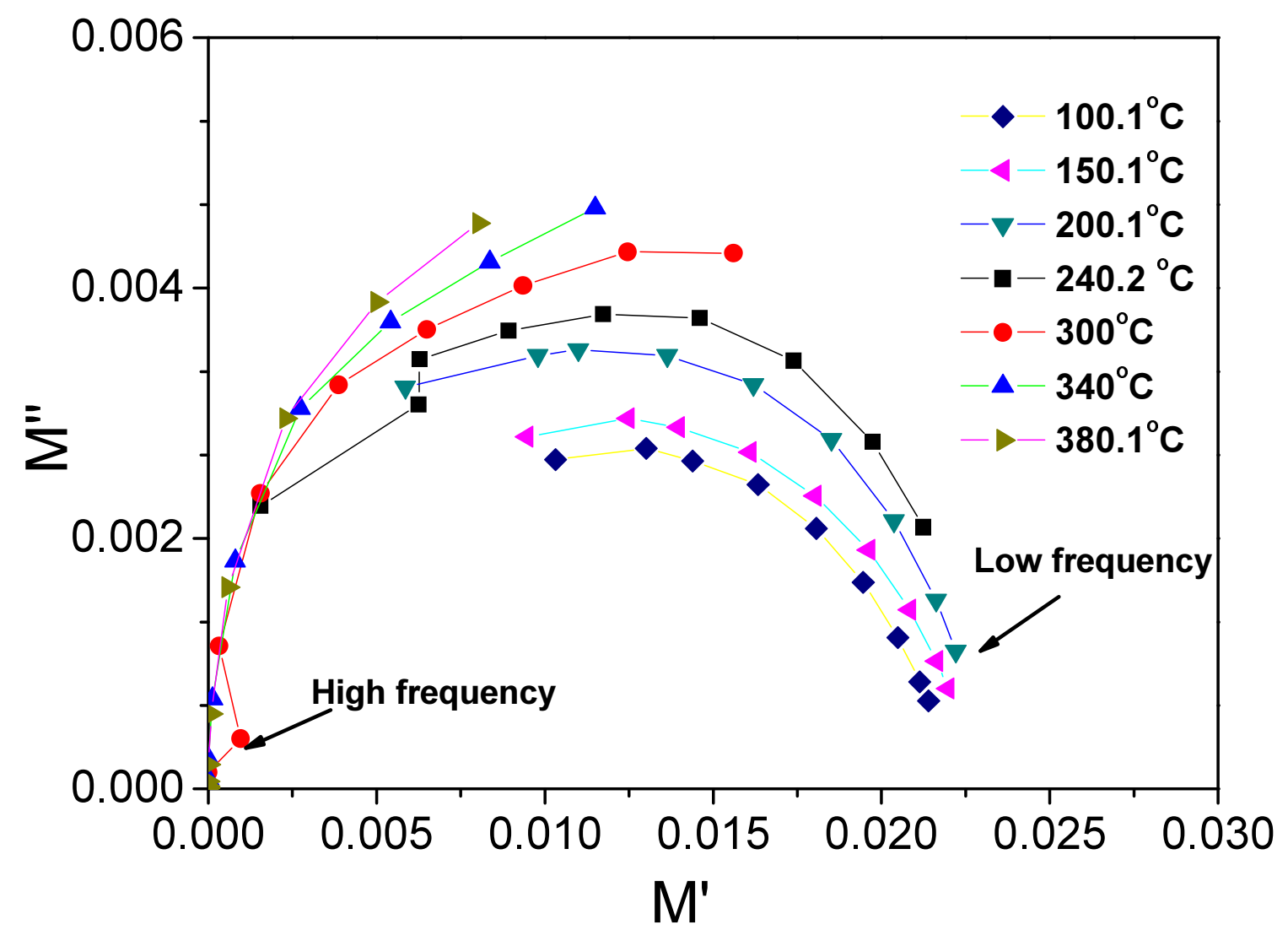

Figure 7 